ARTICLE

\title{
Neighboring Pd single atoms surpass isolated single atoms for selective hydrodehalogenation catalysis
}

\author{
Chiheng Chu1,2,3,8, Dahong Huang 2,3,4,8, Srishti Gupta (1) 3,5, Seunghyun Weon (1) 2,3,6, Junfeng Niu (i) 4 , \\ Eli Stavitski ${ }^{7}$, Christopher Muhich (i) ${ }^{3,5}$ \& $\&$ Jae-Hong Kim (i) $2,3 凶$
}

Single atom catalysts have been found to exhibit superior selectivity over nanoparticulate catalysts for catalytic reactions such as hydrogenation due to their single-site nature. However, improved selectively is often accompanied by loss of activity and slow kinetics. Here we demonstrate that neighboring $\mathrm{Pd}$ single atom catalysts retain the high selectivity merit of sparsely isolated single atom catalysts, while the cooperative interactions between neighboring atoms greatly enhance the activity for hydrogenation of carbon-halogen bonds. Experimental results and computational calculations suggest that neighboring Pd atoms work in synergy to lower the energy of key meta-stable reactions steps, i.e., initial water desorption and final hydrogenated product desorption. The placement of neighboring Pd atoms also contribute to nearly exclusive hydrogenation of carbon-chlorine bond without altering any other bonds in organohalogens. The promising hydrogenation performance achieved by neighboring single atoms sheds light on a new approach for manipulating the activity and selectivity of single atom catalysts that are increasingly studied in multiple applications.

\footnotetext{
${ }^{1}$ Department of Environmental Science, Zhejiang University, Hangzhou, China. ${ }^{2}$ Department of Chemical and Environmental Engineering, Yale University, New Haven, CT, USA. ${ }^{3}$ NSF Nanosystems Engineering Research Center for Nanotechnology Enabled Water Treatment (NEWT), Houston, TX, USA. ${ }^{4}$ School of Environment and Civil Engineering, Dongguan University of Technology, Dongguan, Guangdong, China. ${ }^{5}$ School for the Engineering of Matter, Transport, and Energy, Arizona State University, Tempe, AZ, USA. ${ }^{6}$ School of Health and Environmental Science, Korea University, Seoul, Korea. ${ }^{7}$ National Synchrotron Light Source-II, Brookhaven National Laboratory, Upton, NY, USA. ${ }^{8}$ These authors contributed equally: Chiheng Chu, Dahong Huang.

凶email: Christopher.Muhich@asu.edu; jaehong.kim@yale.edu
} 
T he essential role of heterogeneous catalysis in chemical synthesis, fuel production, and pollutant remediation is driving extensive research efforts in optimizing reactivity and selectivity of catalyst materials. Reducing particle size, often down to nanoscale, and thereby increasing exposure of surface metal atoms have been a common, effective strategy to improve their performance and atom-utilization efficiency ${ }^{1}$. Single atom catalysts (SACs) represent a rapidly emerging trend in catalyst design, where atomic dispersion ensures that each metal atom is exposed and available for catalytic reactions ${ }^{1,2}$. The resultant reduced material usage is particularly attractive for noble metal catalysts that are highly effective in reductive reactions (e.g., hydrogenation) but cost-prohibitive at large scales.

Recent studies suggest that the single-site nature empowers SACs' superior selectivity in select catalytic schemes. Uniform geometric and electronic structure of the catalytic center can favor only one specific adsorption pattern and reaction pathway, resembling that of homogeneous metal catalysts (e.g., ligandbound aqueous phase metal complexes) ${ }^{3}$. This characteristic of SACs is highly desirable in hydrogenation processes that often require selective reduction of the target functional group while keeping the others intact ${ }^{3}$. For instance, highly selective hydrogenation of alkyne 4,5 , diene 6,7 , styrene ${ }^{8}$, nitroarene ${ }^{9}$, and benzaldehyde ${ }^{10}$ has been realized through engineering the geometric configuration of a single metal site that tunes the adsorptivity of reactants/intermediates ${ }^{3}$. However, the high hydrogenation selectivity of SACs is often accompanied by the loss of activity $3,4,6,11$, since the adsorption of a target molecule on a single metal site sterically hindered the adsorption and dissociation of the key reactant, i.e., $\mathrm{H}_{2}{ }^{6}$.

This begs an important question: Can we improve the hydrogenation activity of SACs while maintaining its high selectivity? One potential strategy is to shorten the distance between single atoms such that two neighboring single atoms provide adjacent active sites; this architecture partially resembles nanocatalysts but still maintains the benefit of single atom dispersion. For example, it was recently reported that neighboring Fe single atoms efficiently cleave O-O bonds, a key step in realizing the preferred four-electron oxygen reduction reactions in fuel cells ${ }^{12}$. Because neighboring SACs resemble sparsely isolated SACs (i-SACs) in electronic and geometric structures, they likely retain the catalytic selectivity of i-SACs, while providing higher activity. Nevertheless, there is a dearth of information on which catalytic reactions can benefit from neighboring single atom configurations and a significant knowledge gap in understanding their mechanistic functions.

Here we report the synergistic interactions of neighboring $\mathrm{Pd}$ single atoms that contribute to a superior performance for selective hydrogenation of organohalides. Organohalides occur widely as agrochemicals, pharmaceuticals, solvents, and surfactants, as well as toxic by-products in the effluent of hydraulic fracturing ${ }^{13,14}$. Concerns over the adverse effects of organohalides on the ecosystem and human health have led to rigorous regulations and research needs for developing efficient dehalogenation techniques ${ }^{13}$. We demonstrate that a neighboring Pd SAC $\left(\mathrm{n}-\mathrm{Pd}_{1}\right)$ exhibits both high activity and selectivity for hydrodehalogenation catalysis, significantly surpassing both isolated Pd SAC $\left(\mathrm{i}-\mathrm{Pd}_{1}\right)$ and $\mathrm{Pd}$ nanoparticles $\left(\mathrm{Pd}_{\text {nano }}\right)$. We discuss, based on density functional theory (DFT) calculations, why neighboring single atom configuration is essential for enhanced kinetics and selectivity.

\section{Results and discussion}

Synthesis and structural characterization of Pd SACs. We synthesized Pd SAC by first binding Pd precursors $\left(\mathrm{PdCl}_{4}{ }^{2-}\right)$ onto the amine-modified $\mathrm{SiC}$ surface via electrostatic attraction and subsequently reducing the surface-anchored precursors by a moderate ultraviolet $\mathrm{C}$ (UV-C) irradiation $\left(7.8 \mathrm{~mW} \mathrm{~cm}^{-2}\right)^{10,15}$. Energy-dispersive X-ray spectroscopy suggests successful loading of Pd atoms that are uniformly distributed across the SiC surface (Supplementary Fig. 1). X-ray photoelectron spectroscopy (XPS) elemental analysis (Supplementary Fig. 2) shows that the binding energy of $\mathrm{Pd} 3 \mathrm{~d}_{5 / 2}$ is close to that of $\mathrm{PdCl}_{2}$ reference before UV-C irradiation $(338.0 \mathrm{eV})$ and that of PdO reference after UV-C irradiation $(335.9 \mathrm{eV})$. The atomic dispersion of $\mathrm{Pd}$ and the absence of Pd nanoparticles are confirmed by high-angle annular dark-field scanning transmission electron microscopy (HAADFSTEM) images, where the radius of Pd species is estimated to be $\sim 1.5 \AA$ (Fig. $1 \mathrm{a}-\mathrm{c}$ ). Figure 1a shows that $\mathrm{Pd}$ atoms in $0.5 \%-\mathrm{Pd} / \mathrm{SiC}$ $(\mathrm{Pd}$ mass loading $=0.5 \%)$ are sparsely distributed as i- $\mathrm{Pd}_{1}$. As $\mathrm{Pd}$ loading increases to $1.0 \%$, a small fraction of Pd atoms form groups of neighboring atoms, $\mathrm{n}-\mathrm{Pd}_{1}$ (Fig. $1 \mathrm{~b}$ ), while most remain as $\mathrm{i}-\mathrm{Pd}_{1}$. At $5.6 \%$, most Pd atoms form groups (Fig. 1c) within which neighboring $\mathrm{Pd}$ atoms are randomly distributed. This $n-\mathrm{Pd}_{1}$ distribution pattern is in marked contrast to Pd clusters $\left(\mathrm{Pd}_{\text {nano }}\right)$ that show a much higher $\mathrm{Pd}$ atom density and an ordered crystal structure with clear lattice fringes (Fig. 1d).

The atomic dispersion of $\mathrm{Pd}$ in $0.5 \%-\mathrm{Pd} / \mathrm{SiC}, 1.0 \%-\mathrm{Pd} / \mathrm{SiC}$, and $5.6 \%-\mathrm{Pd} / \mathrm{SiC}$ was confirmed by the absence of Pd-Pd coordination from Fourier-transformed extended X-ray absorption fine structure spectroscopy (FT-EXAFS) analysis at the Pd K-edge (Fig. 1e). Comparison of FT-EXAFS spectra with references indicate that Pd is coordinated with $\mathrm{Cl}$ atoms before UV-C irradiation (Supplementary Fig. 3) and coordinated with $\mathrm{O}$ atoms after UV-C irradiation (Fig. 1e). The distinct Pd-O coordination is in stark contrast with the metal-like Pd-Pd coordination observed in $\mathrm{Pd}_{\text {nano }} / \mathrm{SiC}$ (Fig. 1e). Best-fit parameters extracted from the Pd $K$-edge FT-EXAFS spectra suggest that $0.5 \%-\mathrm{Pd} / \mathrm{SiC}, 1.0 \%-\mathrm{Pd} / \mathrm{SiC}$, and $5.6 \%-\mathrm{Pd} / \mathrm{SiC}$ share a high level of similarity in the first shell structure of Pd single atoms (Supplementary Fig. 4), with an average Pd-O coordination number of 3.2-3.4 and an interatomic distance of 2.03-2.05 nm (Supplementary Table 1). Notably, the second shell Pd-Pd coordination (i.e., peak at 2.4 $\AA$ in FT-EXAFS) of a PdO standard is not observed in $0.5 \%-\mathrm{Pd} / \mathrm{SiC}$ and $1.0 \%-\mathrm{Pd} / \mathrm{SiC}$ (Fig. 1e). In comparison, a small peak at $2.4 \AA$, corresponding to the second shell Pd-Pd coordination, is observed in $5.6 \%-\mathrm{Pd} / \mathrm{SiC}$, confirming the formation of Pd-O-Pd structure in neighboring Pd single atoms. We note that the FTEXAFS pattern of 5.6\%-Pd/SiC is distinct from those of Pd clusters, which exhibit a prominent Pd-Pd coordination with high coordination number (e.g., 6.3-11.0 with Pd cluster sizes of $1.3-10.5 \mathrm{~nm})^{16}$.

$\mathrm{X}$-ray absorption near edge structure (XANES) analysis shows that the white line $(\mathrm{WL})$ intensity of Pd single atoms is between that of $\mathrm{Pd}^{0}$ and $\mathrm{Pd}^{2+}$ (Fig. 1f), suggesting that $\mathrm{Pd}$ atoms are positively charged with partially unoccupied $4 \mathrm{~d}$ orbitals. The first peak of the XANES derivative of $0.5 \%-\mathrm{Pd} / \mathrm{SiC}, 1.0 \%-\mathrm{Pd} / \mathrm{SiC}$, and 5.6\%-Pd/SiC (i.e., peak at 24,348 eV; Fig. $1 \mathrm{~g}$ ) matches that of $\mathrm{PdO}$ and $\mathrm{PdCl}_{2}$ references. This indicates that the valence state of the $\mathrm{Pd}$ single atoms is close to +2 , agreeing with the coordination of Pd single atoms with electrophilic O atoms observed by FTEXAFS spectra (Fig. 1e). The stable Pd oxidation state with increasing loading amount also exclude the formation of $\mathrm{Pd}$ clusters, which, if occurs, should shift Pd oxidation state toward lower value. A significantly higher binding energy of the $\mathrm{Pd} 3 \mathrm{~d}_{5 / 2}$ peak $(335.9 \mathrm{eV})$ compared to that of metallic Pd $(335.3 \mathrm{eV})$ in the XPS spectrum also confirmed the presence of oxidized Pd atoms (Supplementary Fig. 5). DFT calculations confirm that Pd atoms are positively charged, with Bader charges of +0.8 and +0.9 for single atom Pd and PdO reference, respectively. In contrast, the oxidation state of $\mathrm{Pd}$ atoms in $\mathrm{Pd}_{\text {nano }} / \mathrm{SiC}$ is close to that of $\mathrm{Pd}$ foil, as suggested by similar WL intensity (Fig. 1f) and first derivate XANES (first peak at 24,345 eV; Fig. 1g). 

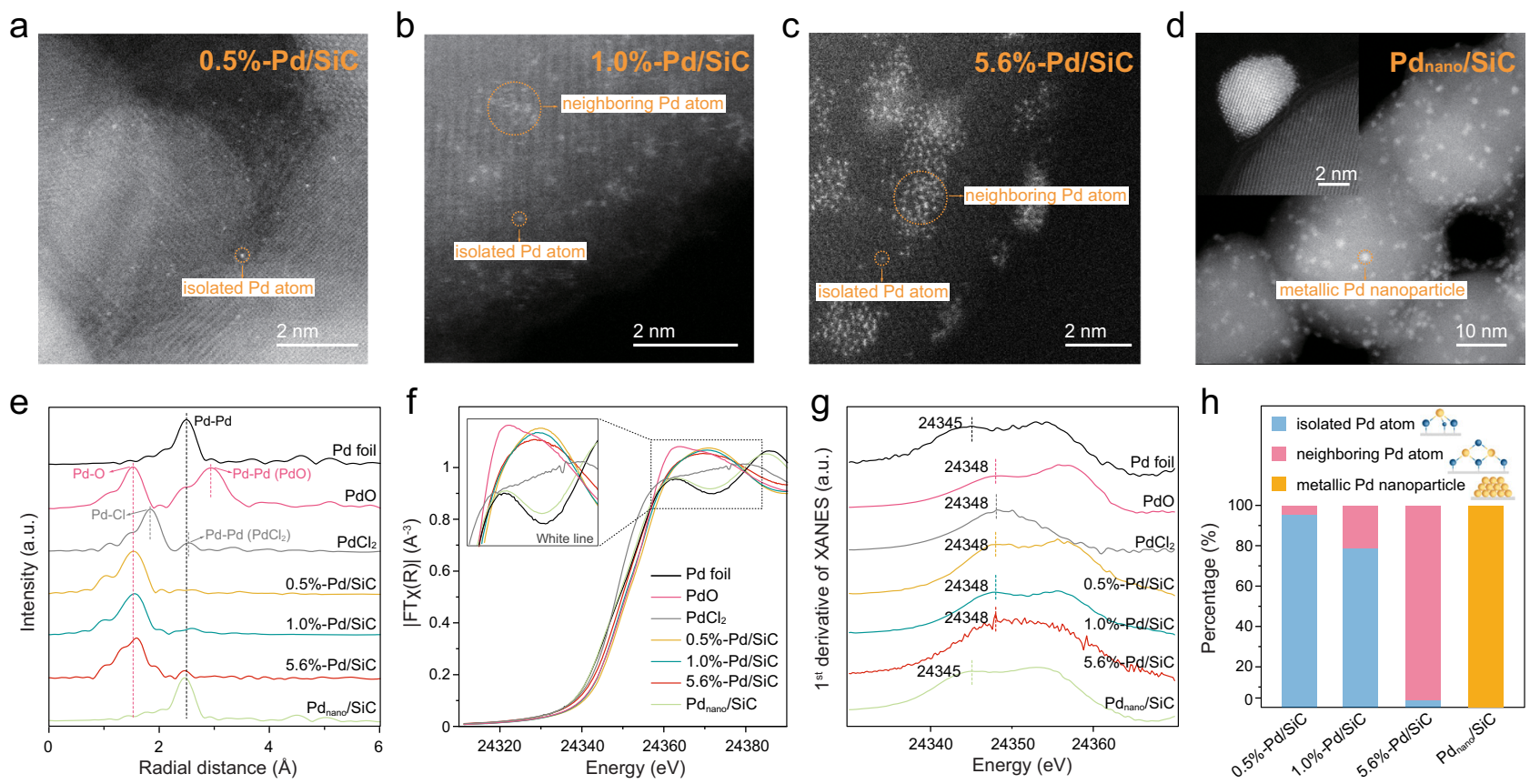

Fig. 1 Structural characterizations of Pd/SiC. a-d High-angle annular dark-field scanning transmission electron microscopy (HAADF-STEM) images of $0.5 \%-\mathrm{Pd} / \mathrm{SiC}, 1.0 \%-\mathrm{Pd} / \mathrm{SiC}, 5.6 \%-\mathrm{Pd} / \mathrm{SiC}$, and $\mathrm{Pd}$ nano/SiC (the inset shows the crystal structure of Pd nanoparticle). e Pd $\mathrm{K}$-edge EXAFS spectra in $R$ space

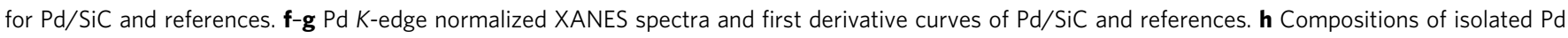
atom, neighboring Pd atom, and metallic Pd nanoparticle.

The above results together suggest that $\mathrm{Pd}$ in $0.5 \%-\mathrm{Pd} / \mathrm{SiC}$, $1.0 \%-\mathrm{Pd} / \mathrm{SiC}$, and $5.6 \%-\mathrm{Pd} / \mathrm{SiC}$ are all in $\mathrm{Pd}_{1}$ state (coordinated with $\mathrm{O}$ atoms and hence positively charged). We define $\mathrm{Pd}$ atoms without a secondary Pd atom in the second shell coordination (i.e., 3.0 and $3.4 \AA$ in $\mathrm{PdO}$ standard) ${ }^{17}$ as i- $\mathrm{Pd}_{1}$; and $\mathrm{Pd}$ atoms interacted with one or more $\mathrm{Pd}$ atoms in the second shell coordination as $\mathrm{n}-\mathrm{Pd}_{1}$. Based on HAADF-STEM images, we estimate that $\mathrm{Pd}$ atoms in $0.5 \%-\mathrm{Pd} / \mathrm{SiC}$ are mostly i- $\mathrm{Pd}_{1}(95.2 \%$, 40 isolated and 2 neighboring $\mathrm{Pd}$ atoms), whereas $1.0 \%-\mathrm{Pd} / \mathrm{SiC}$ consists of both isolated $(80.4 \%, 41$ isolated and 10 neighboring $\mathrm{Pd}$ atoms) and neighboring Pd atoms (19.6\%) (Fig. 1h). Pd atoms in $5.6 \%-\mathrm{Pd} / \mathrm{SiC}$ are dominantly $\mathrm{n}-\mathrm{Pd}_{1}(96.9 \%, 10$ isolated and 313 neighboring Pd atoms).

Hydrodehalogenation activity and selectivity. We tested the aqueous suspension of $\mathrm{Pd} / \mathrm{SiC}$ for catalytic hydrodehalogenation of 4-chlorophenol (4-CP, a model organohalogen compound) under $\mathrm{H}_{2}$ purging at room temperature. To the best of our knowledge, this is the first report where SACs were used to catalyze $\mathrm{H}_{2}$-driven dehalogenation reaction. All $\mathrm{Pd} / \mathrm{SiC}$ materials degraded 4-CP, but they differed in kinetics: $5.6 \%-\mathrm{Pd} / \mathrm{SiC}>$ $\mathrm{Pd}_{\text {nano }} / \mathrm{SiC}>>1.0 \%-\mathrm{Pd} / \mathrm{SiC}>0.5 \%-\mathrm{Pd} / \mathrm{SiC}$ (Fig. $2 \mathrm{a}$ ). The kinetics achieved by $5.6 \%-\mathrm{Pd} / \mathrm{SiC}$ was extremely fast, achieving over $80 \%$ of 4-CP decay within $1 \mathrm{~min}$. The pseudo-first-order rate constant was $2.1 \mathrm{~min}^{-1}$, which is the fastest kinetics ever reported for catalytic 4-CP removal (Supplementary Table 2). Control experiment shows negligible 4-CP degradation on bare $\mathrm{SiC}$ (Supplementary Fig. 6) or by $5.6 \%-\mathrm{Pd} / \mathrm{SiC}$ without $\mathrm{H}_{2}$ purging (Supplementary Fig. 7), confirming the reaction on Pd catalytic centers as the only pathway for $4-\mathrm{CP}$ removal. No significant decreases in the activity of Pd SACs were observed over a 5-cycle of repetitive use (Fig. 2b). XPS (Fig. S5), XANES (Supplementary Fig. 8a), and EXAFS (Supplementary Fig. 8b) analyses show no significant change in chemical composition of $5.6 \%-\mathrm{Pd} / \mathrm{SiC}$ after 1 -week hydrogenation. We attribute the durability of atomically dispersed $\mathrm{Pd}$ to the strong coordination with $\mathrm{O}$ atoms.
Figure $2 \mathrm{c}$ shows that turnover frequency (TOF; per Pd atom basis) of $5.6 \%-\mathrm{Pd} / \mathrm{SiC}$ is 65 times higher than $\mathrm{Pd}_{\text {nanoo }} / \mathrm{SiC}$. This comparison $\left(\mathrm{Pd}_{1}\right.$ versus $\left.\mathrm{Pd}_{\text {nano }}\right)$ highlights the advantage of atomic dispersion, which maximizes the exposure of each $\mathrm{Pd}$ atom as a catalytic center. More interestingly, the TOF dramatically increased at higher Pd loadings: $5.6 \%-\mathrm{Pd} / \mathrm{SiC}>$ $1.0 \%-\mathrm{Pd} / \mathrm{SiC}>0.5 \%-\mathrm{Pd} / \mathrm{SiC}$. A positive correlation between neighboring Pd atom composition and TOF (Fig. 2c) suggests that the activity of $n-\mathrm{Pd}_{1}$ is much higher than that of $\mathrm{i}-\mathrm{Pd}_{1}$. This would not have resulted if all $\mathrm{Pd}_{1}$ atoms catalyzed the reaction at equal efficiency. The result indicates that Pd single atom can more efficiently catalyze the reaction when it has other Pd atoms in its vicinity.

We found that the removal of $4-\mathrm{CP}$ by $\mathrm{Pd} / \mathrm{SiC}$ almost exclusively proceeded through hydrodehalogenation. Results of product analysis (Fig. 2e) suggest that $>99 \%$ selectivity toward dehalogenation (i.e., $>99 \%$ of 4 -CP converted to phenol with concomitant release of $\mathrm{Cl}^{-}$) was achieved by $5.6 \%-\mathrm{Pd} / \mathrm{SiC}$. All $\mathrm{Pd}$ SACs are highly selective toward cleavage of the $\mathrm{C}-\mathrm{Cl}$ bond, with a dehalogenation selectivity $>99 \%$ (Fig. 2d). No significant decrease in 4-CP dehalogenation selectivity was observed within 5 cycles (Fig. 2b). In contrast, much lower dehalogenation selectivity (66\%) was observed in $\mathrm{Pd}_{\text {nano }} / \mathrm{SiC}$ (Fig. 2d), consistent with previous claims that metallic Pd nanoparticles are nonselective between, for example, hydrogenation of conjugated carbons in a benzene ring versus carbons in $\mathrm{C}-\mathrm{Cl}$ bonds ${ }^{18,19}$

Mechanisms of neighboring Pd single atom catalysis. To understand the atomic-level behavior of $\mathrm{i}-\mathrm{Pd}_{1}, \mathrm{n}-\mathrm{Pd}_{1}$, and $\mathrm{Pd}_{\text {nano, }}$, we used DFT to calculate the 4-CP hydrodehalogenation reaction pathways. In this work, we only calculate the meta-stable states along the path. The energy pathways on the various surfaces outlined by the meta-stable state deviate from each other by $>1 \mathrm{eV}$, and the endothermic steps are desorption step, which are normally barrierless. Therefore, the highest reaction barriers that are accessible at experimental conditions would not change the 

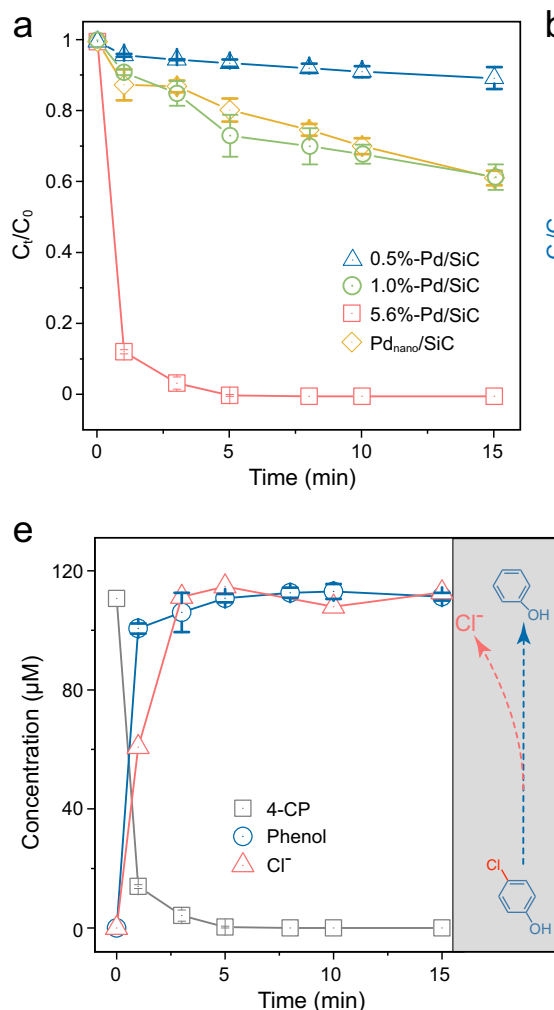
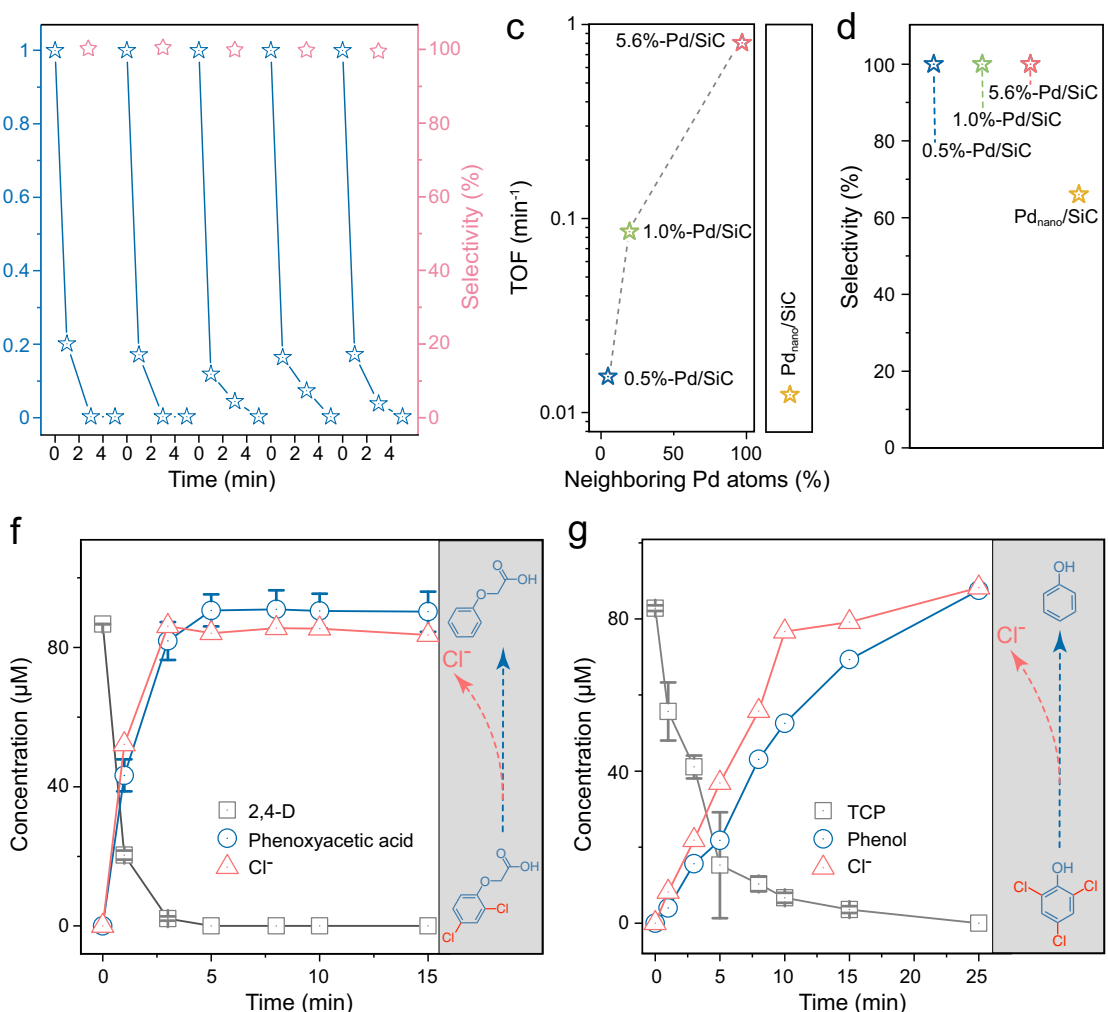

Fig. 2 Catalytic performance of Pd/SiC in the hydrodehalogenation of organohalides. a Hydrodehalogenation kinetics of 4-CP by Pd/SiC. $\mathbf{b}$ Conversion ratio and selectivity of $4-\mathrm{CP}$ at $5 \mathrm{~min}$ by $5.6 \%-\mathrm{Pd} / \mathrm{SiC}$ over five consecutive cycles. c TOF per Pd atom basis calculated with the initial zero-order rate constant of $4-\mathrm{CP}$ hydrogenation. $\mathbf{d}$ Selectivity of hydrodehalogenation. $\mathbf{e}-\mathbf{g}$ Kinetic plots of the organohalide hydrogenation and product generation. The $\mathrm{Cl}^{-}$concentrations in $\mathbf{f}$ and $\mathbf{g}$ were divided by a factor of two (i.e., each 2,4-D molecule contains two $\mathrm{Cl}$ atoms) or three (i.e., each TCP molecule contains three $\mathrm{Cl}$ atoms) to visualize the comparisons. Experimental conditions: catalyst $\left(0.5 \mathrm{~g} \mathrm{~L}^{-1}\right), \mathrm{H}_{2}(1 \mathrm{~atm})$, room temperature $\left(20^{\circ} \mathrm{C}\right)$. Error bars represent standard deviations from triplicate experiments.

conclusions reached here, and we leave detailed transition state analysis to future work.

DFT calculations predict that at least two neighboring Pd atoms are necessary for $\mathrm{C}-\mathrm{Cl}$ bond cleavage, thus explaining the absence of hydrodehalogenation activity on i- $\mathrm{Pd}_{1}$. On $\mathrm{n}-\mathrm{Pd}_{1}$, the cleaved $\mathrm{Cl}^{-}$migrates to the neighboring $\mathrm{Pd}$ atom, stabilizing the anion and providing space for $\mathrm{H}$ to attack the $\mathrm{Pd}$ bound $\mathrm{C}$ (Fig. 3a). This does not occur on i- $\mathrm{Pd}_{1}$ because $\mathrm{Cl}$ does not readily dissociate from the $\mathrm{C}$. In all attempts to force the $\mathrm{Cl}$ away from the $\mathrm{C}$ onto the $\mathrm{i}-\mathrm{Pd}_{1}$ or the $\mathrm{SiC}$ surface, the $\mathrm{Cl}$ relaxed back to the phenol. Therefore, several $\mathrm{H}$ attack routes from the $\mathrm{i}-\mathrm{Pd}_{1}$ and $\mathrm{SiC}$ surface were tested with the $\mathrm{C}-\mathrm{Cl}$ bond intact; in all cases the $\mathrm{H}$ relaxed back onto the $\mathrm{SiC}$ surface, and the $\mathrm{C}-\mathrm{Cl}$ bond remained intact. Hence, hydrodehalogenation reaction on $\mathrm{i}-\mathrm{Pd}_{1}$ is prohibited by steric hindrance and absence of a second metal site for $\mathrm{Cl}$ adsorption prevents further reaction.

We screened several possible reaction paths on the $\mathrm{n}-\mathrm{Pd}_{1}$ and $\mathrm{Pd}_{\text {nano }}$ structures. The lowest energy path proceeds as follows: (1) $\mathrm{H}_{2} \mathrm{O}$ desorption from the $\mathrm{Pd}$, (2) $\mathrm{H}_{2}$ adsorption on $\mathrm{Pd}$, (3) $\mathrm{H}_{2}$ dissociation, (3) $\mathrm{H}$ spillover onto the $\mathrm{SiC}$ or Pd surface, (4) 4-CP adsorption, (5) concerted $\mathrm{S}_{\mathrm{N}} 2$-like $\mathrm{C}-\mathrm{Cl}$ bond cleavage and $\mathrm{C}-\mathrm{H}$ bond formation, and (6) phenol desorption. This reaction path is shown in Fig. 3a. The final $\mathrm{Cl}^{-}$desorption was not included in the analysis as it forms a highly solvated solution anion. We predict that phenol desorption is the rate limiting step on $\mathrm{n}-\mathrm{Pd}_{1}$ $(1.01 \mathrm{eV})$ and on $\mathrm{Pd}_{\text {nano }}(1.82 \mathrm{eV})$, as shown in Fig. 3a. The weaker binding of adsorbates on $\mathrm{n}-\mathrm{Pd}_{1}$ explains its efficient hydrodehalogenation compared to $\mathrm{Pd}_{\text {nano }}$.

The lower barriers for desorption from $\mathrm{n}-\mathrm{Pd}_{1}$ as compared to $\mathrm{Pd}_{\text {nano }}$ stems from weaker individual bond strengths and fewer bonds, which much be broken. The removal of one $\mathrm{H}_{2} \mathrm{O}$ molecule $(1.02 \mathrm{eV})$ from $\mathrm{Pd}_{\text {nano }}$ is more endothermic than on $\mathrm{n}-\mathrm{Pd}_{1}(0.22 \mathrm{eV})$ because the hexagonal $\mathrm{H}$-bonding network of water on the $\mathrm{Pd}_{\text {nano }}$ surface ${ }^{20}$ stabilizes $\mathrm{H}_{2} \mathrm{O}$ adsorption there while only one water can adsorb on $n-\mathrm{Pd}_{1}$. This is further exacerbated because three $\mathrm{H}_{2} \mathrm{O}$ molecules must desorb from $\mathrm{Pd}_{\text {nano }}$ to enable 4-CP adsorption, resulting in the $1.66 \mathrm{eV}$ water desorption barrier. Likewise, the energy barrier for phenol desorption from $\mathrm{n}-\mathrm{Pd}_{1}(1.01 \mathrm{eV})$ is significantly lower than from the $\mathrm{Pd}_{\text {nano }}$ surface $(1.82 \mathrm{eV})$, because only one C-Pd bond breaks instead of six. Overall, the lower energy of phenol and solvent desorption from $\mathrm{n}-\mathrm{Pd}_{1}$ as compared to $\mathrm{Pd}_{\text {nano }}$, results in higher hydrodehalogenation activity of SACs.

We also calculated possible competing reaction paths to $4-\mathrm{CP}$ hydrodehalogenation on $\mathrm{n}-\mathrm{Pd}_{1}$ and $\mathrm{Pd}_{\text {nano }}$ to understand the high selectivity of $\mathrm{n}-\mathrm{Pd}_{1}$. The alternate mechanisms include hydrogenation of the $\mathrm{C}-\mathrm{O}$ bond (hydrodeoxygenation) and hydrogenation of the conjugated carbons in the benzene ring (ring hydrogenation). On $\mathrm{n}-\mathrm{Pd}_{1}, \mathrm{C}-\mathrm{O}$ bond cleavage is $1.37 \mathrm{eV}$ higher in energy than $\mathrm{C}-\mathrm{Cl}$ bond cleavage (Fig. 3b). This corresponds to $\mathrm{C}-\mathrm{O}$ bond cleavage, and thus hydrodeoxygenation, being $10^{-22}$ times less likely to occur than $\mathrm{C}-\mathrm{Cl}$ bond cleavage (step 5) at room temperature based on a Boltzmann distribution. This prevents the hydrogenation of 4-CP proceeding along the deoxygenation path to chlorobenzene (step $6^{*}$ ).

The first step in benzene ring hydrogenation on $\mathrm{n}-\mathrm{Pd}_{1}$ is the migration of a $\mathrm{H}$ from the $\mathrm{C}$ anchored to the $\mathrm{Pd}$ to its neighboring C. This step is endothermic by $1.79 \mathrm{eV}$, which is significantly higher in energy than phenol desorption $(1.01 \mathrm{eV})$. In other words, dissociation of the $\mathrm{C}-\mathrm{Cl}$ bond and hydrogenation of $\mathrm{C}$ on $\mathrm{n}-\mathrm{Pd}_{1}$ is followed by phenol desorption, thus preventing 

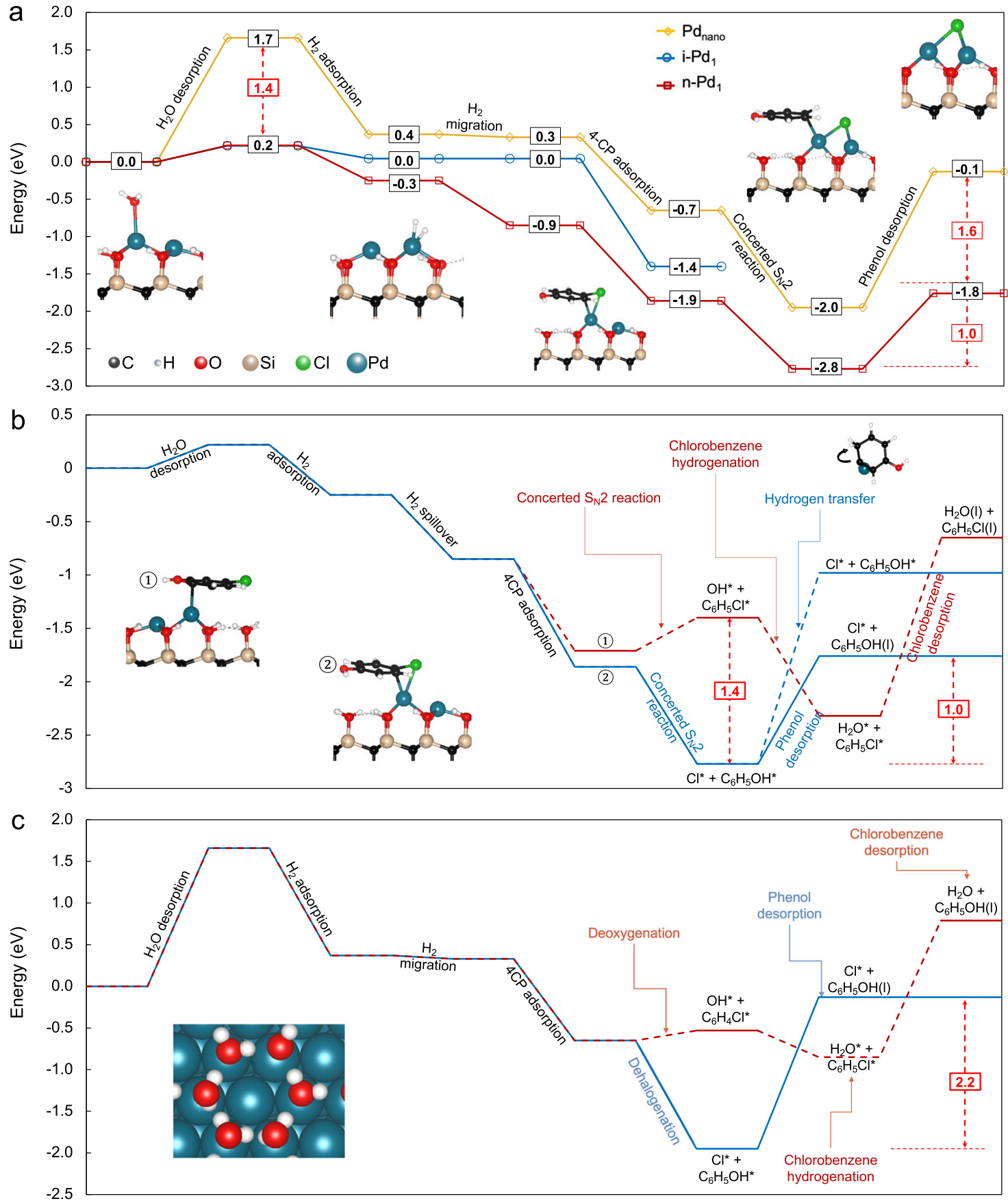

Fig. 3 DFT-calculated minimum energy pathway of 4-CP hydrogenation. a Hydrodehalogenation on $\mathrm{i}-\mathrm{Pd}_{1}, \mathrm{n}-\mathrm{Pd}_{1}$, and $P \mathrm{~d}_{\text {nano. }}$. All steps are exothermic except for $\mathrm{H}_{2} \mathrm{O}$, phenol, and $\mathrm{Cl}^{-}$desorption (not calculated here). b Alternative 4-CP hydrogenation pathways on $\mathrm{n}$ - $\mathrm{Pd} \mathrm{d}_{1}$ (i.e., $\mathrm{C}-\mathrm{O}$ bond cleavage and benzene ring hydrogenation). c Alternative 4-CP hydrogenation pathways on $\mathrm{Pd}_{\text {nano. }}$. The blue, black, red, tan, and white spheres in geometrical models are $\mathrm{Pd}, \mathrm{C}, \mathrm{O}, \mathrm{Si}$, and $\mathrm{H}$ atoms, respectively. The solid and dashed lines represent minimum energy path and other reaction pathways, respectively.

further hydrogenation of the benzene ring. This is in contrast to $\mathrm{Pd}_{\text {nano }}$ where all $\mathrm{C}$ atoms interact with the $\mathrm{Pd}$ surface and are activated, as indicated by the $1.45 \AA$ mean $\mathrm{C}$-C bond length (compared to $1.40 \AA$, mean benzene ring bond length; Fig. $3 \mathrm{c}$ ), meaning that multiple side reactions are possible. These calculations confirm our experimental observation that $\mathrm{n}-\mathrm{Pd}_{1}$ rapidly and selectively cleaves the $\mathrm{C}-\mathrm{Cl}$ bond producing a dehalogenated molecule.

Selective hydrodehalogenation for environmental remediation. While the unprecedented selectivity of $\mathrm{n}-\mathrm{Pd}_{1}$ toward breaking
C-Cl bonds of organohalogens can be exploited for various industrial chemical processes, one application of potentially significant impact is hydrodehalogenation of halogenated contaminants of significant public health concerns, such as 2,4dichlorophenoxyacetic acid (2,4-D; a widely used herbicide and possible human carcinogen) and 2,4,6-trichlorophenol (TCP; a pesticide and possible human carcinogen) in addition to 4-CP discussed above ${ }^{13}$. Note that selectively breaking only $\mathrm{C}-\mathrm{Cl}$ bonds and dehalogenating organics that are polyhalogenated and contain multiple interfering moieties (e.g., ether, ketone) have not been possible with past Pd catalysts. We observed that both 2,4-D and TCP are completely removed in 5 and $15 \mathrm{~min}$, respectively, 
confirming the high catalytic activity of $\mathrm{n}-\mathrm{Pd}_{1}$ in $5.6 \%-\mathrm{Pd} / \mathrm{SiC}$ (Fig. 2f, g). The reduction of polyhalogenated 2,4-D and TCP followed a step-by-step dehalogenation, with the emergence and depletion of mono-chlorinated and di-chlorinated intermediates (Supplementary Figs. 9 and 10). The formation and depletion of dehalogenation intermediates were also evidenced by the changes in $\mathrm{Cl}$ contained in the intermediates (Supplementary Figs. 11 and 12). Phenoxyacetic acid and phenol comprised $>99 \%$ of the final hydrogenation products of 2,4-D (Fig. 2f) and TCP (Fig. 2g); consistently, $>99 \%$ of $\mathrm{Cl}$ atoms in 2,4-D (Fig. 2f) and TCP (Fig. 2g) are recovered as $\mathrm{Cl}^{-}$, further demonstrating the high selectivity of $\mathrm{n}-\mathrm{Pd}_{1}$ for breakage of only $\mathrm{C}-\mathrm{Cl}$ bonds without any side reactions (e.g., hydrogenation of ether, ketone, or benzene moieties in 2,4-D and TCP).

The high hydrodehalogenation selectivity of neighboring $\mathrm{Pd}$ single atoms is also critical for the practical environmental remediation scenarios where competing species are present. For instance, we found that $5.6 \%-\mathrm{Pd} / \mathrm{SiC}$ could also rapidly hydrodehalogenate $4-\mathrm{CP}$ in the presence of natural organic matter $(5 \mathrm{mg}$ $\mathrm{CL}^{-1}$ ), a group of naturally occurring aromatic compounds that typically interfere with the hydrogenation processes in a natural water matrix (Supplementary Fig. 13) ${ }^{21}$. The presence of natural organic matter indeed inhibited 4-CP hydrogenation by $\mathrm{Pd}_{\text {nano }} / \mathrm{SiC}$ due to dominant competing hydrogenation reactions (e.g., benzene ring hydrogenation; Supplementary Fig. 14).

We demonstrate that neighboring Pd single atoms exhibit unprecedently high activity and selectivity for cleavage of the carbon-halogen bond, which are difficult to achieve by either sparsely dispersed single atoms or metallic Pd nanoparticles. Experimental results and computational calculations suggest that at least two neighboring $\mathrm{Pd}$ atoms can synergistically enhance hydrodehalogenation reaction kinetics by lowering the reaction energy of key reactions steps, i.e., initial water desorption and final phenol desorption. The high selectivity of $\mathrm{n}-\mathrm{Pd}_{1}$ originates from significantly lower reaction energy for hydrodehalogenation than other possible reaction pathways, which is in stark contrast to the undifferentiated $\mathrm{H}$ attacks mediated by $\mathrm{Pd}_{\text {nano. This result highlights }}$ that, for the reaction examined in this study and potentially for other reactions, there is plenty of room for catalyst design between isolated single atoms and nanoparticles to achieve higher activity and selectivity. Our findings present a new approach for manipulating the activity and selectivity of SACs that are increasingly studied in a wide range of applications by carefully controlling the metal spatial distribution in nanoscale and sub-nanoscale.

\begin{abstract}
Methods
Catalyst synthesis

Single atom $\mathrm{Pd} / \mathrm{SiC}$. In order to improve the electrostatic adsorption of Pd precursor (i.e., $\mathrm{PdCl}_{4}{ }^{2-}$ ), surface modification of $\mathrm{SiC}$ was conducted by dispersing $0.5 \mathrm{~g} \mathrm{SiC}$ in 250 hexane under ultrasonication for $30 \mathrm{~min}$, followed by the addition of $2 \mathrm{~mL}$ aminopropyltrimethoxysilane and ultrasonication for another $60 \mathrm{~min}$. We note that the surface amines on $\mathrm{SiC}$ also serve to improve catalyst dispersion in water solution during the performance tests (Supplementary Fig. 15). Surfacemodified $\mathrm{SiC}$ was separated by centrifugation, washed with ethanol and deionized water (twice each), and dried at $80^{\circ} \mathrm{C}$ overnight. Following this, $80 \mathrm{mg}$ surfacemodified $\mathrm{SiC}$ was dispersed in $60 \mathrm{~mL}$ deionized water under ultrasonication for $30 \mathrm{~min}$, followed by the addition of $0.38,0.76$, or $4.56 \mathrm{~mL} \mathrm{H}_{2} \mathrm{PdCl}_{4}(10 \mathrm{mM})$. The mixture was stirred for $60 \mathrm{~min}$ and irradiated with UV-C $\left(7.8 \mathrm{~mW} \mathrm{~cm}{ }^{-2}\right)$ for $8 \mathrm{~h}$ at room temperature. As-prepared $\mathrm{Pd} / \mathrm{SiC}$ was washed with water and ethanol (twice each) and dried in a vacuum oven at $80^{\circ} \mathrm{C}$ overnight. The Pd loading amount was determined by inductively coupled plasma mass spectrometry (ICP-MS, PerkinElmer SCIEX Elan DRC-e). Pd atoms did not form direct coordination with amine, as indicated by the unchanged binding energy of Pd in XPS analysis (Supplementary Fig. 16) when amine species were blocked by adding amine capping agent Sulfo-NHS-Acetate (amine-capping evidenced by the dramatic decline in the zeta potential of $5.6 \%-\mathrm{Pd} / \mathrm{SiC}$; Supplementary Fig. 17).
\end{abstract}

$P d_{\text {nano }} / S i C$. Pd nanoparticles were loaded on $\mathrm{SiC}$ through a hydrothermal method by heating suspensions containing $60 \mathrm{mg}$ surface-modified $\mathrm{SiC}(60 \mathrm{mg}), 45.2 \mu \mathrm{mol}$ $\mathrm{H}_{2} \mathrm{PdCl}_{4}$, and $5 \%(\mathrm{v} / \mathrm{v})$ isopropanol as reductant at a heating rate of $10{ }^{\circ} \mathrm{C} \mathrm{min}-1$ to $120^{\circ} \mathrm{C}$ and annealing for $4 \mathrm{~h}$. As-prepared $\mathrm{Pd}_{\text {nano }} / \mathrm{SiC}$ was separated by centrifugation, washed with water and ethanol (twice each), and dried in a vacuum oven at $80^{\circ} \mathrm{C}$ overnight. The Pd loading amount was determined to be $6.2 \%(\mathrm{w} / \mathrm{w})$ by ICP-MS.

Catalyst characterization. HAADF-STEM images were taken on a Titan Themis Z STEM (ThermoFisher Scientific, USA) operated at $200 \mathrm{kV}$, coupled with a probe aberration-corrector to improve imaging spatial resolution to $<1 \AA$. The X-ray absorption spectroscopy spectra at Pd $k$-edge were measured at Beamline 8-ID (ISS) of the National Synchrotron Light Source II at Brookhaven National Laboratory, using a Si (111) double crystal monochromator and a passivated implanted planar silicon fluorescence detector at room temperature, with energy calibrated using Pd foil. The catalyst samples were pressed into a pellet and sealed in Kapton films for XAFS measurements. XAFS data was analyzed using the Athena and Artemis software for conversion of raw data to $\mu(E)$ spectra, background subtraction and normalization, Fourier transformation and plotting, and fitting in $k$-space and $R$-space.

Evaluation of hydrodehalogenation performance. Hydrodehalogenation of organohalogens was performed in a serum glass bottle sealed with rubber septa at room temperature $\left(20^{\circ} \mathrm{C}\right)$. The suspension containing $0.5 \mathrm{~g} \mathrm{~L}^{-1}$ catalyst was purged with $\mathrm{H}_{2}$ (purity $=99.9995 \%$ ) for 5 min prior to the reaction, followed by addition of aqueous stock (5 mM) of 4-CP (Sigma, 99\%), 2,4-D (Sigma, 99\%), or TCP (Sigma, 98\%) using a syringe to initiate the reaction (initial organohalide concentration was $\sim 100 \mu \mathrm{M})$. Aliquots $(250 \mu \mathrm{L})$ were taken at defined time points from the sample solutions by syringe and immediately purged with air to terminate the reactions. To test the catalyst recyclability, 4 -CP was repetitively added up to 5 cycles and aliquots $(150 \mu \mathrm{L})$ were taken at defined time points in each cycle.

Quantification of organohalogens and hydrogenation products. Aliquots were centrifuged and $50 \mu \mathrm{L}$ supernatants was injected into a C18 column at $20^{\circ} \mathrm{C}$, analyzed with an Agilent high-performance liquid chromatography (HPLC) coupled to a photodiode array detector (see Supplementary Table 3 for detailed HPLC methods), and quantified with corresponding analytical standards. For quantification of $\mathrm{Cl}^{-}$, the $\mathrm{pH}$ of aliquots was adjusted to $\sim 13$ by adding $\mathrm{NaOH}(1 \mathrm{M})$ to detach $\mathrm{Cl}^{-}$from the positively charged $\mathrm{SiC}$ surface. Afterwards, the aliquots were centrifuged and $100 \mu \mathrm{L}$ supernatants were removed, diluted, and injected into a Dionex ion chromatography, and quantified with corresponding analytical standards.

Computational methodology. Periodic boundary condition DFT calculations were performed at the Perdew-Burke-Ernzerhof level, as implemented in the Vienna Ab initio Simulation Program (VASP) ${ }^{22-24}$. Non-local van der Waals interactions were accounted for through the DFT-D3 correction, which is based on the method of Grimme et al. ${ }^{25}$. The wave function was formed from a summation of plane waves with energies $<500 \mathrm{eV}$. We used the projected-augmented wave method pseudopotentials ${ }^{26}$ to reduce the number of plane waves required, where we explicitly included the $\mathrm{H} 1 \mathrm{~s}, \mathrm{O}$, and $\mathrm{C} 2 \mathrm{~s} 2 \mathrm{p}, \mathrm{Cl} 3 \mathrm{~s} 3 \mathrm{p}$, and Pd 5s4d electrons.

$\mathrm{Pd}$ and single/neighboring atom Pd catalyst surfaces were repressed by a Pd slab consisting of a supercell with a (111) terminated $4 \times 4 \times 6$ primitive units and a single and neighboring Pd atoms on a $(001)$ terminated $(6 \times 6 \times 4)$ Si-C slab, respectively. The surface terminations were chosen because they represent the lowenergy surfaces ${ }^{27-29}$. Both slabs were separated by at least $12 \AA$ of vacuum space. The Brillion zone was sampled on a $6 \times 6 \times 1$ and $2 \times 2 \times 1 \Gamma$-point centered Mohnkorst Pack mesh for the Pd and $\mathrm{SiC}$ surfaces, respectively. A layer of $\mathrm{H}_{2} \mathrm{O}$ and dissociated $\mathrm{H}_{2} \mathrm{O}(\mathrm{OH})$ covered the Pd surface and $\mathrm{SiC}$ surface, respectively, to mimic the water environment from the experiments. The Pd atoms catalysts were bonded to the SiC surface via two surface oxygen (Fig. 1b). All geometries were relaxed to $1.0 \times 10^{-4} \mathrm{eV}$. The energies of molecules in water are calculated with implicit solvent using the VASPsol module ${ }^{30,31}$. Geometric structure representations were generated using VESTA. The activation barriers for the exothermic steps were not calculated, as they are likely to be lower in energy than those for endothermic desorption.

\section{Data availability}

The data that support the findings of this study are available from the corresponding authors upon reasonable request.

Received: 25 March 2020; Accepted: 3 August 2021; Published online: 30 August 2021

\section{References}

1. Wang, A., Li, J. \& Zhang, T. Heterogeneous single-atom catalysis. Nat. Rev. Chem. 2, 65-81 (2018). 
2. Yang, X. F. et al. Single-atom catalysts: a new frontier in heterogeneous catalysis. Acc. Chem. Res. 46, 1740-1748 (2013).

3. Zhang, L., Zhou, M., Wang, A. \& Zhang, T. Selective hydrogenation over supported metal catalysts: from nanoparticles to single atoms. Chem. Rev. 120, 683-733 (2019).

4. Huang, F. et al. Atomically dispersed Pd on nanodiamond/graphene hybrid for selective hydrogenation of acetylene. J. Am. Chem. Soc. 140, 13142-13146 (2018).

5. Vile, G. et al. A Stable single-site palladium catalyst for hydrogenations. Angew. Chem. Int. Ed. 54, 11265-11269 (2015).

6. Yan, H. et al. Understanding the underlying mechanism of improved selectivity in pd1 single-atom catalyzed hydrogenation reaction. J. Catal. 366, 70-79 (2018).

7. Yan, H. et al. Single-atom $\mathrm{Pd}(1)$ /graphene catalyst achieved by atomic layer deposition: remarkable performance in selective hydrogenation of 1,3butadiene. J. Am. Chem. Soc. 137, 10484-10487 (2015).

8. Kyriakou, G. et al. Isolated metal atom geometries as a strategy for selective heterogeneous hydrogenations. Science 335, 1209-1212 (2012).

9. Lin, L. et al. A highly CO-tolerant atomically dispersed Pt catalyst for chemoselective hydrogenation. Nat. Nanotechnol. 14, 354-361 (2019).

10. Liu, P. X. et al. Photochemical route for synthesizing atomically dispersed palladium catalysts. Science 352, 797-801 (2016).

11. Jorgensen, M. \& Gronbeck, H. Selective acetylene hydrogenation over singleatom alloy nanoparticles by kinetic Monte Carlo. J. Am. Chem. Soc. 141, 8541-8549 (2019).

12. Chen, W. et al. Neighboring Pt atom sites in an ultrathin FePt nanosheet for the efficient and highly CO-tolerant oxygen reduction reaction. Nano Lett. 18, 5905-5912 (2018).

13. Alonso, F., Beletskaya, I. P. \& Yus, M. Metal-mediated reductive hydrodehalogenation of organic halides. Chem. Rev. 102, 4009-4092 (2002).

14. Richardson, S. D. \& Ternes, T. A. Water analysis: emerging contaminants and current issues. Anal. Chem. 90, 398-428 (2018).

15. Huang, D. et al. Single-atom Pt catalyst for effective C-F bond activation via hydrodefluorination. ACS Catal. 8, 9353-9358 (2018).

16. Vogel, W., Knözinger, H., Carvill, B. T., Sachtler, W. M. H. \& Zhang, Z. C. Mass diffusion effect in large zeolite $\mathrm{Y}$ aggregates on Pd cluster size distribution: a combined in situ EXAFS/XRD study. J. Phys. Chem. B. 102, 1750-1758 (1998).

17. Chu, C. et al. Electronic tuning of metal nanoparticles for highly efficient photocatalytic hydrogen peroxide production. ACS Catal. 9, 626-631 (2019).

18. Brieger, G. \& Nestrick, T. J. Catalytic transfer hydrogenation. Chem. Rev. 74, 567-580 (1974).

19. Johnstone, R. A. W., Wilby, A. H. \& Entwistle, I. D. Heterogeneous catalytic transfer hydrogenation and its relation to other methods for reduction of organic compounds. Chem. Rev. 85, 129-170 (1985).

20. Cerda, J. et al. Novel water overlayer growth on $\operatorname{Pd}(111)$ characterized with scanning tunneling microscopy and density functional theory. Phys. Rev. Lett. 93, 116101-116104 (2004)

21. Kopinke, F.-D., Angeles-Wedler, D., Fritsch, D. \& Mackenzie, K. Pd-catalyzed hydrodechlorination of chlorinated aromatics in contaminated waters-effects of surfactants, organic matter and catalyst protection by silicone coating. Appl. Catal. B Environ. 96, 323-328 (2010).

22. Kresse, G. \& Furthmüller, J. Efficient iterative schemes for ab initio totalenergy calculations using a plane-wave basis set. Phys. Rev. B 54, 11169-11186 (1996).

23. Kresse, G. \& Furthmüller, J. Efficiency of ab-initio total energy calculations for metals and semiconductors using a plane-wave basis set. Comp. Mater. Sci. 6, 15-50 (1996).

24. Perdew, J. P., Burke, K. \& Ernzerhof, M. Generalized gradient approximation made simple. Phys. Rev. Lett. 77, 3865-3868 (1996).

25. Grimme, S., Antony, J., Ehrlich, S. \& Krieg, H. A consistent and accurate ab initio parametrization of density functional dispersion correction (DFT-D) for the 94 elements H-Pu. J. Chem. Phys. 132, 154104-154122 (2010).

26. Kresse, G. \& Joubert, D. From ultrasoft pseudopotentials to the projector augmented-wave method. Phys. Rev. B 59, 1758-1775 (1999).

27. Catellani, A., Galli, G. \& Gygi, F. Reconstruction and thermal stability of the cubic SiC (001) surfaces. Phys. Rev. Lett. 77, 5090-5093 (1996).
28. Vitos, L., Ruban, A. V., Skriver, H. L. \& Kollar, J. The surface energy of metals. Surf. Sci. 411, 186-202 (1998).

29. Wen, Y. N. \& Zhang, H. M. Surface energy calculation of the fcc metals by using the MAEAM. Solid State Commun. 144, 163-167 (2007).

30. Mathew, K., Kolluru, V. S. C., Mula, S., Steinmann, S. N. \& Hennig, R. G. Implicit self-consistent electrolyte model in plane-wave density-functional theory. J. Chem. Phys. 151, 234101 (2019).

31. Mathew, K., Sundararaman, R., Letchworth-Weaver, K., Arias, T. A. \& Hennig, R. G. Implicit solvation model for density-functional study of nanocrystal surfaces and reaction pathways. J. Chem. Phys. 140, 084106 (2014).

\section{Acknowledgements}

We thank the National Science Foundation Nanosystems Engineering Research Center for Nanotechnology-Enabled Water Treatment (EEC-1449500) for partial support. We are grateful to J. Karosas (Yale) for help with ICP-MS, and P. Kelleher (BNL) for help in sample pellet preparation for XAFS measurements. This research used beamline 8-ID (ISS) of the National Synchrotron Light Source II, U.S. Department of Energy (DOE) Office of Science User Facilities operated for the DOE Office of Science by Brookhaven National Laboratory under Contract No. DE-SC0012704. Computational work used the Extreme Science and Engineering Discovery Environment (XSEDE), supported by NSF (ACI-1548562), through the Bridges high-performance computer at the Pittsburgh Supercomputing Center (allocation ECD190001).

\section{Author contributions}

C.C., D.H., C.M., and J.-H.K. designed research; C.C. and D.H. synthesized the catalysts and conducted performance test; S.G. and C.M. performed DFT calculations. C.C., D.H., S.W., and E.S. conducted XAFS measurements; D.H. and J.N. conducted STEM measurements. C.C., D.H., S.G., E.S., C.M., and J.-H.K. analyzed the data; and C.C., S.G., C.M., and J.-H.K. wrote the paper. All authors discussed the results and commented on the manuscript.

\section{Competing interests}

The authors declare no competing interests.

\section{Additional information}

Supplementary information The online version contains supplementary material available at https://doi.org/10.1038/s41467-021-25526-2.

Correspondence and requests for materials should be addressed to C.M. or J.-H.K.

Peer review information Nature Communications thanks the anonymous reviewers for their contribution to the peer review of this work.

Reprints and permission information is available at http://www.nature.com/reprints

Publisher's note Springer Nature remains neutral with regard to jurisdictional claims in published maps and institutional affiliations.

Open Access This article is licensed under a Creative Commons Attribution 4.0 International License, which permits use, sharing, adaptation, distribution and reproduction in any medium or format, as long as you give appropriate credit to the original author(s) and the source, provide a link to the Creative Commons license, and indicate if changes were made. The images or other third party material in this article are included in the article's Creative Commons license, unless indicated otherwise in a credit line to the material. If material is not included in the article's Creative Commons license and your intended use is not permitted by statutory regulation or exceeds the permitted use, you will need to obtain permission directly from the copyright holder. To view a copy of this license, visit http://creativecommons.org/ licenses/by/4.0/

(C) The Author(s) 2021 